\title{
On Mean Square Stability and Dissipativity of Split-Step Theta Method for Nonlinear Neutral Stochastic Delay Differential Equations
}

\author{
Haiyan Yuan, ${ }^{1,2}$ Jihong Shen, ${ }^{1}$ and Cheng Song ${ }^{3}$ \\ ${ }^{1}$ College of Automation, Harbin Engineering University, Harbin 150001, China \\ ${ }^{2}$ Department of Mathematics, Heilongjiang Institute of Technology, Harbin 150050, China \\ ${ }^{3}$ School of Management, Harbin Institute of Technology, Harbin 150001, China \\ Correspondence should be addressed to Haiyan Yuan; yhy82_47@163.com
}

Received 2 March 2016; Revised 6 June 2016; Accepted 23 June 2016

Academic Editor: Guang Zhang

Copyright ( 2016 Haiyan Yuan et al. This is an open access article distributed under the Creative Commons Attribution License, which permits unrestricted use, distribution, and reproduction in any medium, provided the original work is properly cited.

\begin{abstract}
A split-step theta (SST) method is introduced and used to solve the nonlinear neutral stochastic delay differential equations (NSDDEs). The mean square asymptotic stability of the split-step theta (SST) method for nonlinear neutral stochastic delay differential equations is studied. It is proved that under the one-sided Lipschitz condition and the linear growth condition, the split-step theta method with $\theta \in(1 / 2,1]$ is asymptotically mean square stable for all positive step sizes, and the split-step theta method with $\theta \in[0,1 / 2]$ is asymptotically mean square stable for some step sizes. It is also proved in this paper that the split-step theta (SST) method possesses a bounded absorbing set which is independent of initial data, and the mean square dissipativity of this method is also proved.
\end{abstract}

\section{Introduction}

Stochastic functional differential equations (SFDEs) play important roles in science and engineering applications, especially for systems whose evolutions in time are influenced by random forces as well as their history information. When the time delays in SFDEs are constants, they turn into stochastic delay differential equations (SDDEs). Both the theory and numerical methods for SDDEs have been well developed in the recent decades; see [1-8]. Recently, many dynamical systems not only depend on the present and the past states but also involve derivatives with delays; they are described as the neutral stochastic delay differential equations (NSDDEs). Compared to the stochastic differential equations and the stochastic delay differential equations, the study of the neutral stochastic delay differential equations has just started. In 1981, Kolmanovskii and Myshkis [9] took the environmental disturbances into account, introduced the neutral stochastic delay differential equations (NSDDEs), and gave their applications in chemical engineering and aeroelasticity. The analytical solutions of NSDDEs are hard to obtain; many authors have to study the numerical methods for NSDDEs. Wu and Mao [10] studied the convergence of the Euler-Maruyama method for neutral stochastic functional differential equations under the one-side Lipschitz conditions and the linear growth conditions. In 2009, Zhou and Wu [11] studied the convergence of the Euler-Maruyama method for NSDDEs with Markov switching under the one-side Lipschitz conditions and the linear growth conditions. The convergence of $\theta$-method and the mean square asymptotic stability of the semi-implicit Euler method for NSDDEs were studied by Gan et al. [12], Zhou and Fang [13], and Yin and Ma [14], respectively. Later, the almost sure exponential stability of Euler-Maruyama method for NSDDEs was studied in [15] with the discrete semimartingale convergence theorem.

To the best of our knowledge, most of these studies have focused on the convergence of numerical solutions for NSDDEs; the stability and dissipativity of numerical solutions for them are rarely concerned.

The aim of this paper is to study the mean square stability and dissipativity of the split-step theta method with some conditions and the step constrained for NSDDEs. 
The paper is organized as follows. In Section 2, some stability definitions about the analytic solutions for NSDDEs are introduced; some notations and preliminaries are also presented in this section. In Section 3, the split-step theta method is introduced and used to solve the NSDDEs; the asymptotic stability of the split-step theta method is proved. In Section 4, the long time behavior of numerical solution is studied and the mean square dissipativity result of the method is illustrated. In Section 5, some numerical experiments are given to confirm the theoretical results.

\section{Exponential Mean Square Stability of Analytic Solution}

Let $|\cdot|$ denote both the Euclidean norm in $R^{d}$ and the trace (or Frobenius) norm in $R^{d \times l}$ (denoted by $|A|=$ $\left.\sqrt{\operatorname{trace}\left(A^{\mathrm{T}} A\right)}\right)$; if $A$ is a vector or matrix, its transpose is denoted by $A^{\mathrm{T}}$. Let $\left\{\Omega, F,\left\{F_{t}\right\}_{t \geq 0}, \mathrm{P}\right\}$ define a complete probability space with a filtration $\left\{F_{t}\right\}_{t \geq 0}$ which is increasing and right continuous, and $F_{0}$ contain all P-null sets. Let $w(t)=\left(w_{1}(t), w_{2}(t), \ldots, w_{l}(t)\right)^{\mathrm{T}}$ denote standard $l$ dimensional Brownian motion on the probability space. In this paper we talk about the $d$-dimensional NSDDEs with the following form:

$$
\begin{aligned}
& d(y(t)-N(y(t-\tau))) \\
& =f(t, y(t), y(t-\tau)) d t \\
& \quad+g(t, y(t), y(t-\tau)) d w(t), \quad t \geq 0, \\
& \quad y(t)=\varphi(t), \quad t \in[-\tau, 0],
\end{aligned}
$$

where $N: R^{d} \mapsto R^{d}, f: R_{+} \times R^{d} \times R^{d} \mapsto R^{d}$, and $g: R_{+} \times R^{d} \times R^{d} \mapsto R^{d \times l}$ are the Borel measurable functions. $\tau$ is a positive constant delay, and $\varphi(t)$ is $F_{0}$-measurable, $C\left([-\tau, 0] ; R^{d}\right)$-valued random variable which satisfies

$$
\sup _{-\tau \leq t \leq 0} \mathrm{E}\left[\varphi^{\mathrm{T}}(t) \varphi(t)\right]<+\infty
$$

with the notation E denoting the mathematical expectation with respect to $P$.

The following conditions (a1) and (a2) are standard for the existence and uniqueness of the solution for (1).

(a1) The Local Lipschitz Condition. There exist constants $K_{L}>$ 0 and $L>0$ such that

$$
\begin{aligned}
& \left|f\left(t, x_{1}, y_{1}\right)-f\left(t, x_{2}, y_{2}\right)\right|^{2} \\
& \quad \vee\left|g\left(t, x_{1}, y_{1}\right)-g\left(t, x_{2}, y_{2}\right)\right|^{2} \\
& \leq K_{L}\left(\left|x_{1}-x_{2}\right|^{2}+\left|y_{1}-y_{2}\right|^{2}\right)
\end{aligned}
$$

for all $\left|x_{1}\right| \vee\left|x_{2}\right| \vee\left|y_{1}\right| \vee\left|y_{2}\right| \leq L$ and $t \in R_{+}$, where $a \vee b$ represents $\max \{a, b\}$ and $a \wedge b$ represents $\min \{a, b\}$.

(a2) The Linear Growth Condition. There exists a constant $K_{G}>0$, such that

$$
\begin{aligned}
& |f(t, x, y)|^{2} \vee|g(t, x, y)|^{2} \vee|N(x)|^{2} \\
& \quad \leq K_{G}\left(1+|x|^{2}+|y|^{2}\right)
\end{aligned}
$$

for all $(t, x, y) \in R_{+} \times R \times R$.

As an especial case of Theorem 3.1 in Mao's monograph (see [6]), we can easily know that under hypothesis (a1) and (a2), system (1) has a global unique continuous solution on $t \geq-\tau$, which is denoted by $y(t)$.

Now we recall some stability concepts for the solution of (1).

Definition 1 (see [6]). The trivial solution of (1) is said to be exponentially mean square stable, if there exists a pair of constants $r>0$ and $C>0$, such that, whenever $\sup _{-\tau \leq t \leq 0} \mathrm{E}\left[\varphi^{\mathrm{T}}(t) \varphi(t)\right]<+\infty$,

$$
\mathrm{E}\left[y^{\mathrm{T}}(t) y(t)\right] \leq C \sup _{-\tau \leq t \leq 0} \mathrm{E}\left[\varphi^{\mathrm{T}}(t) \varphi(t)\right] e^{-r t}, \quad t \geq 0 .
$$

Lemma 2. Assume that there exist a symmetric, positive definite $d \times d$ matrix $Q$ and positive constants $\mu_{1}, \mu_{2}$, and $\lambda \in(0,1)$ such that for all $(t, x, y) \in R_{+} \times R^{d} \times R^{d}$

$$
\begin{aligned}
& |N(x)| \leq \lambda|x|, \\
& (x-N(y))^{\mathrm{T}} Q f(t, x, y) \\
& \quad+\frac{1}{2} \operatorname{trace}\left[g^{\mathrm{T}}(t, x, y) Q g(t, x, y)\right] \leq-\mu_{1} x^{\mathrm{T}} Q x \\
& \quad+\mu_{2} y^{\mathrm{T}} Q y .
\end{aligned}
$$

If conditions

$$
\begin{gathered}
0<\lambda<\frac{1}{2}, \\
\mu_{1}>\frac{\mu_{2}}{(1-2 \lambda)^{2}}
\end{gathered}
$$

hold, then the trivial solution of (1) is exponentially mean square stable.

Remark 3. In general, we require $\lambda \neq 0$. When $\lambda=0$, (1) becomes a stochastic delay differential equation. Many stability and dissipativity results have been studied in the literature (see $[5,16])$.

By Lemma 2, the following result can easily be obtained.

Theorem 4. Suppose (6) holds. Assume that there are positive constants $\lambda_{1}, \lambda_{2}$, and $K$, such that, for all $x, y \in R^{d}$,

$$
\begin{aligned}
(x-N(y))^{\mathrm{T}} \mathrm{Q} f(t, x, y) \leq-\lambda_{1} x^{\mathrm{T}} \mathrm{Q} x+\lambda_{2} y^{\mathrm{T}} \mathrm{Q} y, \\
|f(t, x, y)|^{2} \vee|g(t, x, y)|^{2} \leq K\left(|x|^{2}+|y|^{2}\right) .
\end{aligned}
$$


If conditions

$$
\begin{aligned}
0 & <\lambda<\frac{1}{2}, \\
\lambda_{1} & >\frac{1}{2} K+\frac{2 \lambda_{2}+K}{2(1-2 \lambda)^{2}}
\end{aligned}
$$

hold, then the trivial solution of (1) is exponentially mean square stable.

Proof. Consider (9) and the inequality; we get the following inequality:

$$
\begin{aligned}
(x & -N(y))^{\mathrm{T}} Q f(t, x, y) \\
& +\frac{1}{2} \operatorname{trace}\left[g^{\mathrm{T}}(t, x, y) Q g(t, x, y)\right] \\
\leq & -\lambda_{1} x^{\mathrm{T}} \mathrm{Q} x+\lambda_{2} y^{\mathrm{T}} \mathrm{Q} y+\frac{1}{2} K\left(x^{\mathrm{T}} Q x+y^{\mathrm{T}} \mathrm{Q} y\right) \\
\leq & -\left(\lambda_{1}-\frac{1}{2} K\right) x^{\mathrm{T}} Q x+\left(\lambda_{2}+\frac{1}{2} K\right) \mu_{2} y^{\mathrm{T}} Q y .
\end{aligned}
$$

Let $\mu_{1}=\left(\lambda_{1}-(1 / 2) K\right), \mu_{2}=\left(\lambda_{2}+(1 / 2) K\right)$; when conditions (10) hold, we get that

$$
\mu_{1}>\frac{\mu_{2}}{(1-2 \lambda)^{2}}
$$

Using Lemma 2, we can easily prove that the trivial solution of (1) is exponentially mean square stable.

\section{The Stability of the Split-Step Theta Method}

The split-step theta method is proved to be able to keep the mean square asymptotic stability of the exact solution under the sufficient conditions of the asymptotic stability of the exact solution, so in this paper we use the split-step theta method to solve the NSDDE.

Appling the split-step theta (SST) method into problem (1) gives the following form:

$$
\begin{aligned}
Y_{n}-N Y_{n-m}= & y_{n}-N y_{n-m} \\
& +\theta \Delta t f\left(t_{n}+\theta \Delta t, Y_{n}, \bar{Y}_{n}\right), \\
\bar{Y}_{n}= & Y_{n-m}, \\
y_{n+1}-N y_{n+1-m}= & y_{n}-N y_{n-m} \\
& +\Delta t f\left(t_{n}+\theta \Delta t, Y_{n}, \bar{Y}_{n}\right) \\
& +g\left(t_{n}+\theta \Delta t, Y_{n}, \bar{Y}_{n}\right) \Delta w_{n},
\end{aligned}
$$

where the step size $\Delta t=\tau / m, m$ is an integer, $y_{i}$ is an approximation to $y\left(t_{i}\right), t_{i}=i \Delta t, i=1,2, \ldots$, and $y_{k}=$ $Y_{k}=\varphi(k \Delta t)$ for $k=-m,-m+1, \ldots, 0 . \theta \in[0,1]$ is a fixed parameter, and $\Delta w_{k}:=w((k+1) \Delta t)-w(k \Delta t)$ is the Brownian increment.

When $\theta=0$ the split-step theta method is simplified into the split-step forward Euler method and when $\theta=1$ the split-step theta method is simplified into the split-step backward Euler method. They were discussed for stochastic differential equations in [17-20]. In order to consider the stability property of scheme (13)-(15) we should give some stability concepts for numerical methods firstly.

Definition 5 (see [16]). For a given step size $\Delta t$, a numerical method is said to be exponentially mean square stable if there is a pair of positive constants $\gamma$ and $C$ such that for any initial data $\varphi(t)$ the numerical solution $y_{n}$ produced by the method satisfies

$$
\mathrm{E}\left[y_{n}^{\mathrm{T}} y_{n}\right] \leq C e^{-\gamma t_{n}} \sup _{-\tau \leq t \leq 0} \mathrm{E}\left[\varphi^{\mathrm{T}}(t) \varphi(t)\right], \quad \forall n \geq 0
$$

Definition 6 (see [16]). For a given step size $\Delta t$, a numerical method is said to be asymptotically mean square stable if for any initial data $\varphi(t)$ the numerical solution $y_{n}$ produced by the method satisfies

$$
\lim _{n \rightarrow \infty} \mathrm{E}\left[y_{n}^{\mathrm{T}} y_{n}\right]=0
$$

Theorem 7. Assume that system (1) satisfies (7) with $-\mu_{1}+$ $\mu_{2}<0$; then the SST method (13)-(15) with $\theta \in(1 / 2,1]$ is asymptotically mean square stable for all $\Delta t>0$. If we further assume that there exist constants $K_{1}$ and $K_{2}$ such that

$$
\begin{array}{r}
f^{\mathrm{T}}(t, x, y) Q f(t, x, y) \leq K_{1} x^{\mathrm{T}} Q x+K_{2} y^{\mathrm{T}} Q y, \\
\quad(t, x, y) \in R_{+} \times R^{d} \times R^{d},
\end{array}
$$

then, for any $\theta \in[0,1 / 2)$, there exists a constant $\Delta t_{0}$ depending on $\theta$ such that the method is asymptotically mean square stable for $\Delta t \in\left(0, \Delta t_{0}\right)$.

Proof. From (15) it follows that

$$
\begin{aligned}
& \left(y_{n+1}-N y_{n+1-m}\right)^{\mathrm{T}} \mathrm{Q}\left(y_{n+1}-N y_{n+1-m}\right) \\
& \quad=\left(y_{n}-N y_{n-m}\right)^{\mathrm{T}} \mathrm{Q}\left(y_{n}-N y_{n-m}\right) \\
& +\Delta t^{2} f^{\mathrm{T}}\left(t_{n}+\theta \Delta t, Y_{n}, \bar{Y}_{n}\right) Q f\left(t_{n}+\theta \Delta t, Y_{n}, \bar{Y}_{n}\right) \\
& +\Delta w_{n}^{\mathrm{T}} g^{\mathrm{T}}\left(t_{n}+\theta \Delta t, Y_{n}, \bar{Y}_{n}\right) Q g\left(t_{n}+\theta \Delta t, Y_{n}, \bar{Y}_{n}\right) \\
& \cdot \Delta w_{n}+2\left(y_{n}-N y_{n-m}\right)^{\mathrm{T}} \Delta t Q f\left(t_{n}+\theta \Delta t, Y_{n}, \bar{Y}_{n}\right) \\
& +2\left(y_{n}-N y_{n-m}\right)^{\mathrm{T}} \mathrm{Qg}\left(t_{n}+\theta \Delta t, Y_{n}, \bar{Y}_{n}\right) \Delta w_{n} \\
& +2 \Delta t f^{\mathrm{T}}\left(t_{n}+\theta \Delta t, Y_{n}, \bar{Y}_{n}\right) Q g\left(t_{n}+\theta \Delta t, Y_{n}, \bar{Y}_{n}\right) \\
& +\Delta w_{n} .
\end{aligned}
$$


Since $w(t)=\left(w_{1}(t), w_{2}(t), \ldots, w_{l}(t)\right)^{\mathrm{T}}$ is a standard $l$-dimensional Brownian motion we have that

$$
\begin{aligned}
& \mathrm{E}\left(\Delta w_{i}\right)=0, \\
& \mathrm{E}\left[\left(\Delta w_{i}\right)^{2}\right]=\Delta t, \\
& \mathrm{E}\left[\Delta w_{n}^{\mathrm{T}} g^{\mathrm{T}}\left(t_{n}+\theta \Delta t, Y_{n}, \bar{Y}_{n}\right) \mathrm{Qg}\left(t_{n}+\theta \Delta t, Y_{n}, \bar{Y}_{n}\right)\right. \\
& \left.\cdot \Delta w_{n}\right]=\Delta t \mathrm{E}\left[\operatorname{trace}^{\mathrm{T}}\left(t_{n}+\theta \Delta t, Y_{n}, \bar{Y}_{n}\right)\right. \\
& \left.\cdot \mathrm{Qg}\left(t_{n}+\theta \Delta t, Y_{n}, \bar{Y}_{n}\right)\right] .
\end{aligned}
$$

Let $x_{n}=y_{n}-N y_{n-m}, X_{n}=Y_{n}-N Y_{n-m}, n=0,1, \ldots$, substitute the designation into (19) and then, taking expectation on both sides, one receives

$$
\begin{aligned}
\mathrm{E}\left[x_{n+1}^{\mathrm{T}} \mathrm{Q} x_{n+1}\right] \leq \mathrm{E}\left[x_{n}^{\mathrm{T}} \mathrm{Q} x_{n}\right]+(1-2 \theta) \Delta t^{2} f^{\mathrm{T}}\left(t_{n}\right. \\
\left.+\theta \Delta t, Y_{n}, \bar{Y}_{n}\right) Q f\left(t_{n}+\theta \Delta t, Y_{n}, \bar{Y}_{n}\right)+2 \Delta t \mathrm{E}\left(Y_{n}\right. \\
\left.-N Y_{n-m}\right)^{\mathrm{T}} \Delta t \mathrm{Q} f\left(t_{n}+\theta \Delta t, Y_{n}, \bar{Y}_{n}\right) \\
+\Delta t \mathrm{E}\left[\operatorname{traceg}^{\mathrm{T}}\left(t_{n}+\theta \Delta t, Y_{n}, \bar{Y}_{n}\right)\right. \\
\left.\cdot \operatorname{Qg}\left(t_{n}+\theta \Delta t, Y_{n}, \bar{Y}_{n}\right)\right],
\end{aligned}
$$

which, combined with (7), gives

$$
\begin{aligned}
& \mathrm{E}\left[x_{n+1}^{\mathrm{T}} \mathrm{Q} x_{n+1}\right] \leq \mathrm{E}\left[x_{n}^{\mathrm{T}} \mathrm{Q} x_{n}\right] \\
& +2 \Delta t \mathrm{E}\left(-\mu_{1} Y_{n}^{\mathrm{T}} \mathrm{Q} Y_{n}+\mu_{2} \bar{Y}_{n}^{\mathrm{T}} \mathrm{Q} \bar{Y}_{n}\right)+(1-2 \theta) \\
& \cdot \Delta t^{2} f^{\mathrm{T}}\left(t_{n}+\theta \Delta t, Y_{n}, \bar{Y}_{n}\right) Q f\left(t_{n}+\theta \Delta t, Y_{n}, \bar{Y}_{n}\right) .
\end{aligned}
$$

In the case of $\theta>1 / 2$, using

$$
\begin{aligned}
& \Delta t f\left(t_{n}+\theta \Delta t, Y_{n}, \bar{Y}_{n}\right)=\frac{1}{\theta}\left(X_{n}-x_{n}\right), \\
& 2 X_{n}^{\mathrm{T}} Q x_{n} \\
& \leq \frac{2 \theta-1-\left(-\mu_{1}+\mu_{2}\right) \Delta t \theta^{2}}{2 \theta-1} X_{n}^{\mathrm{T}} Q X_{n} \\
& \quad+\frac{2 \theta-1}{2 \theta-1-\left(-\mu_{1}+\mu_{2}\right) \Delta t \theta^{2}} x_{n}^{\mathrm{T}} Q x_{n},
\end{aligned}
$$

then we have

$$
\begin{aligned}
& \mathrm{E}\left[x_{n+1}^{\mathrm{T}} \mathrm{Q} x_{n+1}\right] \\
& \leq\left(1+\frac{\left(-\mu_{1}+\mu_{2}\right) \Delta t(2 \theta-1)}{2 \theta-1-\left(-\mu_{1}+\mu_{2}\right) \Delta t \theta^{2}}\right) \mathrm{E}\left[x_{n}^{\mathrm{T}} Q x_{n}\right] \\
&-2 \Delta t \mu_{2} \mathrm{E}\left[Y_{n}^{\mathrm{T}} \mathrm{QY} Y_{n}\right] \\
&+2 \Delta t\left(\left(1-\lambda^{2}\right) \mu_{2}+\lambda^{2} \mu_{1}\right) \mathrm{E}\left[\bar{Y}_{n}^{\mathrm{T}} \mathrm{Q} \bar{Y}_{n}\right] .
\end{aligned}
$$

Let

$$
\begin{gathered}
k=\max \left\{1+\frac{\left(-\mu_{1}+\mu_{2}\right) \Delta t(2 \theta-1)}{2 \theta-1-\left(-\mu_{1}+\mu_{2}\right) \Delta t \theta^{2}},\right. \\
\left.\left(\frac{\mu_{2}}{\left(\left(1-\lambda^{2}\right) \mu_{2}+\lambda^{2} \mu_{1}\right)}\right)^{1 / m}\right\} ;
\end{gathered}
$$

we can deduce that $0<k<1$.

By induction, the following results are obtained from (24):

$$
\begin{aligned}
& \mathrm{E}\left[x_{n+1}^{\mathrm{T}} \mathrm{Q} x_{n+1}\right] \\
& \leq k^{n+1} \mathrm{E}\left[x_{0}^{\mathrm{T}} \mathrm{Q} x_{0}\right]-2 \Delta t \mu_{2} \sum_{j=0}^{n} k^{n-j} \mathrm{E}\left[Y_{j}^{\mathrm{T}} \mathrm{Q} Y_{j}\right] \\
& \quad+2 \Delta t\left(\left(1-\lambda^{2}\right) \mu_{2}+\lambda^{2} \mu_{1}\right) \sum_{j=0}^{n} k^{n-j} \mathrm{E}\left[\bar{Y}_{j}^{\mathrm{T}} \mathrm{Q} \bar{Y}_{j}\right] .
\end{aligned}
$$

Using condition (14), we can get the following inequality:

$$
\begin{aligned}
\sum_{j=0}^{n} k^{n-j} \mathrm{E}\left[\bar{Y}_{j}^{\mathrm{T}} Q \bar{Y}_{j}\right] \leq & m k^{n-m+1} \max _{-m \leq j \leq-1} \mathrm{E}\left[Y_{j}^{\mathrm{T}} Q Y_{j}\right] \\
& +k^{-m} \sum_{j=0}^{n-m+1} k^{n-j} \mathrm{E}\left[Y_{j}^{\mathrm{T}} Q Y_{j}\right] .
\end{aligned}
$$

Therefore,

$$
\begin{aligned}
\mathrm{E}[ & \left.x_{n+1}^{\mathrm{T}} \mathrm{Q} x_{n+1}\right] \leq k^{n+1}\left(\mathrm{E}\left[x_{0}^{\mathrm{T}} \mathrm{Q} x_{0}\right]\right. \\
& \left.+2 \tau\left(\left(1-\lambda^{2}\right) \mu_{2}+\lambda^{2} \mu_{1}\right) k^{-m} \max _{-m \leq j \leq-1} \mathrm{E}\left[Y_{j}^{\mathrm{T}} \mathrm{Q} Y_{j}\right]\right) \\
& -2 \Delta t\left(\mu_{2}-\left(\left(1-\lambda^{2}\right) \mu_{2}+\lambda^{2} \mu_{1}\right) k^{-m}\right) \\
& \cdot \sum_{j=0}^{n-m+1} k^{n-j} \mathrm{E}\left[Y_{j}^{\mathrm{T}} \mathrm{Q} Y_{j}\right] .
\end{aligned}
$$

It can be deduced from (28) and (25) that $-\left(\mu_{2}-\left(\left(1-\lambda^{2}\right) \mu_{2}+\right.\right.$ $\left.\left.\lambda^{2} \mu_{1}\right) k^{-m}\right) \leq 0$, so, we can have the following inequality:

$$
\begin{aligned}
& \mathrm{E}\left[x_{n+1}^{\mathrm{T}} \mathrm{Q} x_{n+1}\right] \leq k^{n+1}\left(\mathrm{E}\left[x_{0}^{\mathrm{T}} \mathrm{Q} x_{0}\right]\right. \\
& \left.\quad+2 \tau\left(\left(1-\lambda^{2}\right) \mu_{2}+\lambda^{2} \mu_{1}\right) k^{-m} \max _{-m \leq j \leq-1} \mathrm{E}\left[Y_{j}^{\mathrm{T}} \mathrm{Q} Y_{j}\right]\right) .
\end{aligned}
$$

On the other hand, we know that

$$
\begin{aligned}
\left\|y_{n+1}\right\| & =\left\|y_{n+1}-N y_{n+1-m}+N y_{n+1-m}\right\| \\
& \leq\left\|x_{n+1}\right\|+\left\|N y_{n+1-m}\right\|,
\end{aligned}
$$

then we get

$$
\begin{aligned}
\mathrm{E}\left[y_{n+1}^{\mathrm{T}} Q y_{n+1}\right] \leq & 2 \mathrm{E}\left[x_{n+1}^{\mathrm{T}} \mathrm{Q} x_{n+1}\right] \\
& +2 \lambda^{2} \mathrm{E}\left[y_{n+1-m}^{\mathrm{T}} \mathrm{Q} y_{n+1-m}\right] ;
\end{aligned}
$$


define

$$
\begin{aligned}
\varepsilon_{0} & =k^{n+1}\left(\mathrm{E}\left[x_{0}^{\mathrm{T}} \mathrm{Q} x_{0}\right]+2 \tau\left(\left(1-\lambda^{2}\right) \mu_{2}+\lambda^{2} \mu_{1}\right) k^{-m}\right. \\
& \left.\cdot \max _{-m \leq j \leq-1} \mathrm{E}\left[Y_{j}^{\mathrm{T}} \mathrm{Q} Y_{j}\right]\right)
\end{aligned}
$$

the following inequality could be deduced from (31):

$$
\begin{aligned}
\mathrm{E}\left[y_{n+1}^{\mathrm{T}} \mathrm{Q} y_{n+1}\right] \leq & \frac{2}{1-2 \lambda^{2}} \varepsilon_{0} \\
& +\left(2 \lambda^{2}\right)^{\lfloor n / m\rfloor+1} \max _{-m \leq j \leq-1} \mathrm{E}\left[y_{j}^{\mathrm{T}} \mathrm{Q} y_{j}\right],
\end{aligned}
$$

which implies that the method is asymptotically mean square stable.

For the case that $\theta \in[0,1 / 2)$, with the hypothesis (18) and (22) we can obtain the following inequality:

$$
\begin{aligned}
\mathrm{E}\left[x_{n+1}^{\mathrm{T}} \mathrm{Q} x_{n+1}\right] \\
\leq \mathrm{E}\left[x_{n}^{\mathrm{T}} \mathrm{Q} x_{n}\right] \\
\quad+\Delta t\left((1-2 \theta) \Delta t K_{1}-2 \mu_{1}\right) \mathrm{E}\left[Y_{n}^{\mathrm{T}} \mathrm{Q} Y_{n}\right] \\
\quad+\Delta t\left((1-2 \theta) \Delta t K_{2}+2 \mu_{2}\right) \mathrm{E}\left[\bar{Y}_{n}^{\mathrm{T}} \mathrm{Q} \bar{Y}\right] .
\end{aligned}
$$

A combination of (13) and (18) gives

$$
x_{n}^{\mathrm{T}} \mathrm{Q} x_{n} \leq L_{1} Y_{n}^{\mathrm{T}} \mathrm{Q} Y_{n}+L_{2} \bar{Y}_{n}^{\mathrm{T}} \mathrm{Q} \bar{Y}_{n},
$$

where $L_{1}=(1+\theta \Delta t)\left(2+\theta \Delta t K_{1}\right), L_{2}=(1+\theta \Delta t)\left(2 \lambda^{2}+\theta \Delta t K_{2}\right)$.

Let

$$
\Delta t_{0}= \begin{cases}+\infty, & \theta=\frac{1}{2}, \\ \frac{-2\left(-\mu_{1}+\mu_{2}\right)}{(1-2 \theta)\left(K_{1}+K_{2}\right)}, & \theta \in\left[0, \frac{1}{2}\right) ;\end{cases}
$$

then, for any fixed $\Delta t \in\left(0, \Delta t_{0}\right), 2\left(-\mu_{1}+\mu_{2}\right)+\Delta t(1-2 \theta)\left(K_{1}+\right.$ $\left.K_{2}\right)<0$, there exists a small positive number $\varepsilon$ such that

$$
\begin{aligned}
& 2\left(-\mu_{1}+\mu_{2}\right)+\Delta t(1-2 \theta)\left(K_{1}+K_{2}\right)+\frac{L_{1}+L_{2}}{\Delta t} \varepsilon \\
& \quad<0 .
\end{aligned}
$$

Therefore,

$$
\begin{aligned}
\mathrm{E}\left[x_{n+1}^{\mathrm{T}} \mathrm{Q} x_{n+1}\right] \\
\leq(1-\varepsilon) \mathrm{E}\left[x_{n}^{\mathrm{T}} \mathrm{Q} x_{n}\right] \\
\quad+\Delta t\left((1-2 \theta) \Delta t K_{1}-2 \mu_{1}+\frac{L_{1}}{\Delta t} \varepsilon\right) \mathrm{E}\left[Y_{n}^{\mathrm{T}} \mathrm{Q} Y_{n}\right] \\
\quad+\Delta t\left((1-2 \theta) \Delta t K_{2}+2 \mu_{2}+\frac{L_{2}}{\Delta t} \varepsilon\right) \mathrm{E}\left[\bar{Y}_{n}^{\mathrm{T}} \mathrm{Q} \bar{Y}_{n}\right]
\end{aligned}
$$

Let $\widetilde{k}=\max \left\{1-\varepsilon,\left(\left((1-2 \theta) \Delta t K_{2}+2 \mu_{2}+\left(L_{2} / \Delta t\right) \varepsilon\right) /-((1-\right.\right.$ $\left.\left.\left.2 \theta) \Delta t K_{1}-2 \mu_{1}+\left(L_{1} / \Delta t\right) \varepsilon\right)\right)^{1 / m}\right\}$; then $0<\tilde{k}<1$. Similar to the derivation of the first part, the following inequality can be proved from (38):

$$
\begin{aligned}
& \mathrm{E}\left[x_{n+1}^{\mathrm{T}} \mathrm{Q} x_{n+1}\right] \\
& \quad \leq k^{n+1}\left(\mathrm{E}\left[x_{0}^{\mathrm{T}} \mathrm{Q} x_{0}\right]+\widetilde{L} \tilde{k}^{-m} \max _{-m \leq j \leq-1} \mathrm{E}\left[Y_{j}^{\mathrm{T}} \mathrm{Q} Y_{j}\right]\right),
\end{aligned}
$$

where $\widetilde{L}=\tau\left((1-2 \theta) \Delta t K_{2}+2 \mu_{2}+\left(L_{2} / \Delta t\right) \varepsilon\right)$.

Similar to the proof of (31), we can prove that when $\Delta t \epsilon$ $\left(0, \Delta t_{0}\right)$ the method is asymptotically mean square stable; the proof of theorem is completed.

Remark 8. For system (1) with $N=0$, it becomes a stochastic delay differential equation; the mean square stability of the theta method has been studied in [16]; Theorem 7 can be regarded as an extension of Theorem 3.4 presented in [16].

Remark 9. For the NSDDEs, the mean square asymptotic stability of the BEM method has been studied by Wang and Chen in [15]; it has shown that BEM method can reproduce the mean square stability of the exact solutions; Theorem 7 improves the result in [15].

\section{Mean Square Dissipativity}

The numerical solutions' long time dynamic behavior will be studied in this section. Before it, we make the following hypothesis: assume that there exist a symmetric, positive definite $d \times d$ matrix $Q$ and positive constants $\mu_{1}, \mu_{2}$, and $\gamma$ such that, for all $(t, x, y) \in R_{+} \times R^{d} \times R^{d}$, the following inequality exists:

$$
\begin{aligned}
{[x-} & N(y)]^{\mathrm{T}} Q f(t, x, y) \\
& +\frac{1}{2} \operatorname{trace}\left[g^{\mathrm{T}}(t, x, y) Q g(t, x, y)\right] \\
\leq & \gamma-\mu_{1} x^{\mathrm{T}} \mathrm{Q} x+\mu_{2} y^{\mathrm{T}} \mathrm{Q} y .
\end{aligned}
$$

Now we state and prove some conclusions.

Definition 10 (see [16]). Assume that system (1) satisfies (40). The numerical method is said to be dissipative if when the method is applied to problem (1) with constraint $\tau=m h$, there exists a constant $C$ such that, for any initial values, there exists $n_{0}$, depending only on initial values $\varphi(t)$, such that

$$
\mathrm{E}\left[y_{n}^{\mathrm{T}} \mathrm{Q} y_{n}\right] \leq C, \quad n \geq n_{0}
$$

Theorem 11. Assume that system (1) satisfies (40); there exists a constant $C$ such that, for any initial values, there exists $n_{0}$ depending only on the initial values $\varphi(t)$, when $n \geq n_{0}$, the numerical solution $y_{n}$ generated by the SST method (13)-(15) with $\theta \in(1 / 2,1]$, such that

$$
\mathrm{E}\left[y_{n}^{\mathrm{T}} \mathrm{Q} y_{n}\right] \leq C
$$


Proof. Consider (21) and (40); the following inequality can be obtained:

$$
\begin{aligned}
& \mathrm{E}\left[x_{n+1}^{\mathrm{T}} \mathrm{Q} x_{n+1}\right] \leq \mathrm{E}\left[x_{n}^{\mathrm{T}} \mathrm{Q} x_{n}\right]+2 \Delta t \gamma \\
& \quad+2 \Delta t \mathrm{E}\left(-\mu_{1} Y_{n}^{\mathrm{T}} \mathrm{Q} Y_{n}+\mu_{2} \bar{Y}_{n}^{\mathrm{T}} \mathrm{Q} \bar{Y}_{n}\right)+(1-2 \theta) \\
& \cdot \Delta t^{2} f^{\mathrm{T}}\left(t_{n}+\theta \Delta t, Y_{n}, \bar{Y}_{n}\right) \mathrm{Q} f\left(t_{n}+\theta \Delta t, Y_{n}, \bar{Y}_{n}\right) .
\end{aligned}
$$

We can get the following inequality the same as the derivation of (28):

$$
\begin{aligned}
& \mathrm{E}\left[x_{n+1}^{\mathrm{T}} \mathrm{Q} x_{n+1}\right] \leq k^{n+1}\left(\mathrm{E}\left[x_{0}^{\mathrm{T}} \mathrm{Q} x_{0}\right]\right. \\
&+\left.2 \tau\left(\left(1-\lambda^{2}\right) \mu_{2}+\lambda^{2} \mu_{1}\right) k^{-m} \max _{-m \leq j \leq-1} \mathrm{E}\left[Y_{j}^{\mathrm{T}} \mathrm{Q} Y_{j}\right]\right) \\
&-2 \Delta t\left(\mu_{2}-\left(\left(1-\lambda^{2}\right) \mu_{2}+\lambda^{2} \mu_{1}\right) k^{-m}\right) \\
& \cdot \sum_{j=0}^{n-m+1} k^{n-j} \mathrm{E}\left[Y_{j}^{\mathrm{T}} \mathrm{Q} Y_{j}\right]+2 \Delta t \gamma \sum_{j=0}^{n} k^{j},
\end{aligned}
$$

where $0<k<1$ is the same as defined in (25).

Because $-\left(\mu_{2}-\left(\left(1-\lambda^{2}\right) \mu_{2}+\lambda^{2} \mu_{1}\right) k^{-m}\right) \leq 0$, we have the following inequality:

$$
\begin{aligned}
& \mathrm{E}\left[x_{n+1}^{\mathrm{T}} \mathrm{Q} x_{n+1}\right] \leq k^{n+1}\left(\mathrm{E}\left[x_{0}^{\mathrm{T}} \mathrm{Q} x_{0}\right]\right. \\
& \left.+2 \tau\left(\left(1-\lambda^{2}\right) \mu_{2}+\lambda^{2} \mu_{1}\right) k^{-m} \max _{-m \leq j \leq-1} \mathrm{E}\left[Y_{j}^{\mathrm{T}} \mathrm{Q} Y_{j}\right]\right) \\
& +\frac{2 \gamma \Delta t}{1-k} .
\end{aligned}
$$

Let

$$
\begin{aligned}
\varepsilon_{1} & =k^{n+1}\left(\mathrm{E}\left[x_{0}^{\mathrm{T}} \mathrm{Q} x_{0}\right]+2 \tau\left(\left(1-\lambda^{2}\right) \mu_{2}+\lambda^{2} \mu_{1}\right) k^{-m}\right. \\
& \left.\cdot \max _{-m \leq j \leq-1} \mathrm{E}\left[Y_{j}^{\mathrm{T}} \mathrm{Q} Y_{j}\right]\right)+\frac{2 \gamma \Delta t}{1-k} ;
\end{aligned}
$$

the following inequality could be deduced from (31):

$$
\begin{aligned}
\mathrm{E}\left[y_{n+1}^{\mathrm{T}} \mathrm{Q} y_{n+1}\right] \leq & 2 \mathrm{E}\left[x_{n+1}^{\mathrm{T}} \mathrm{Q} x_{n+1}\right] \\
& +2 \lambda^{2} \mathrm{E}\left[y_{n+1-m}^{\mathrm{T}} \mathrm{Q} y_{n+1-m}\right] \\
\leq & 2 \varepsilon_{1}+2 \lambda^{2} \mathrm{E}\left[y_{n+1-m}^{\mathrm{T}} \mathrm{Q} y_{n+1-m}\right] \leq C,
\end{aligned}
$$

where $C=2 \varepsilon_{1} /\left(1-2 \lambda^{2}\right)+\varepsilon$. The theorem is completed.

Theorem 11 means that the discrete system possesses a bounded absorbing set in the sense of mean square. The numerical solution trajectory from any initial date will enter the set in a finite time and thereafter remain inside. It is called mean square dissipativity.

Remark 12. For the study of the dissipativity of numerical methods for deterministic delay differential equations with constant delays, Huang and Chang studied the dissipativity of Runge-Kutta methods and multistep Runge-Kutta methods in $[21,22]$.

\section{The Numerical Experiment}

In this section, we will give a numerical experiment to illustrate the stability and dissipativity result obtained in Sections 3 and 4 . Consider the following nonlinear scalar neutral stochastic delay differential equation:

$$
\begin{aligned}
& d[y(t)-0.25 \sin (y(t-1))] \\
& \quad=[-8 y(t)+\sin (y(t-1))] d t+y(t-1) d W(t), \\
& t \geq 0, \\
& y(t)=t+1, \quad-1 \leq t \leq 0 .
\end{aligned}
$$

It is easy to verify that nonlinear neutral stochastic delay differential equation (48) satisfies the conditions of Theorem 7; the corresponding parameters are given as follows:

$$
\begin{aligned}
\lambda & =\frac{1}{4}, \\
\mu_{1} & =8, \\
\mu_{2} & =\frac{1}{2}, \\
K_{1} & =64, \\
K_{2} & =1, \\
\Delta t_{0} & =\left\{\begin{array}{l}
+\infty, \quad \theta=\frac{1}{2} \\
0.2884, \quad \theta \in\left(0, \frac{1}{2}\right) .
\end{array}\right.
\end{aligned}
$$

The initial condition is given by $y(t)=t+1, t \in$ $[-1,0]$, where we take $\tau=1$. In the following tests, we show the influence of step size $\Delta t$ and the parameter $\theta$ on M-S stability of the SST method; the data used in all figures are obtained by the mean square of data by 200 trajectories; that is, $\mathrm{E} y_{n}^{2} \approx(1 / 200) \sum_{i=1}^{200}\left[y_{n}^{(i)}\right]^{2}, \mathrm{E} y_{n} \approx(1 / 200) \sum_{i=1}^{200} y_{n}^{(i)}$, where $y_{n}^{(i)}$ denotes the numerical solution of $y\left(t_{n}\right)$ in the $i$ th trajectory.

Taking step sizes $\Delta t=0.1, \Delta t=0.2, \Delta t=0.3$, and $\Delta t=0.6$, we obtain the numerical solutions of (48), and the numerical solutions are displayed in Figures 1-6, respectively. We can see that when $\theta=0.6$, the SST method is asymptotically mean square stable for all the step sizes selected, but when $\theta=0.1$, the SST method is asymptotically mean square stable only for the step sizes $\Delta t \leq 0.2884$; it is not mean square stable for the step sizes $\Delta t>0.2884$.

\section{Competing Interests}

The authors declare that there is no conflict of interests regarding the publication of this paper. 


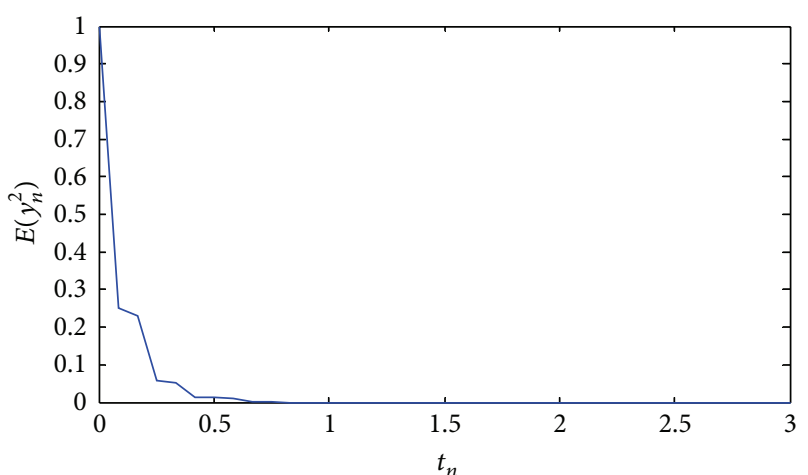

FIGURE 1: Mean square stability of SST method with $\theta=0.6$ and $\Delta t=0.1$.

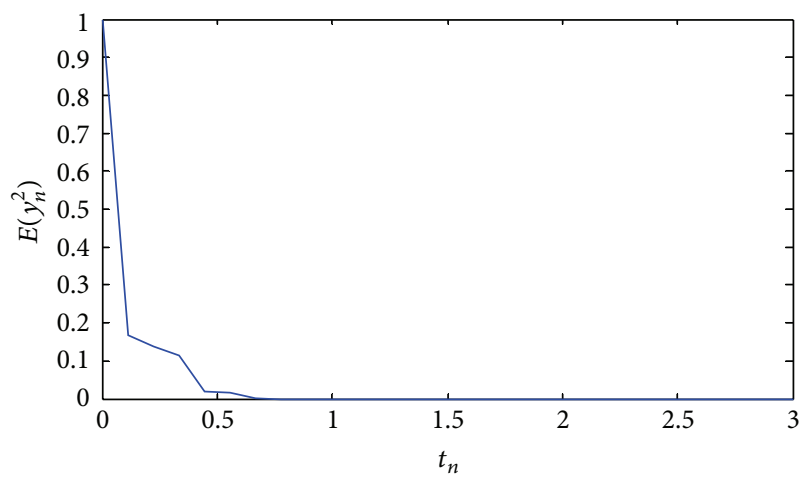

FIGURE 2: Mean square stability of SST method with $\theta=0.6$ and $\Delta t=0.6$.

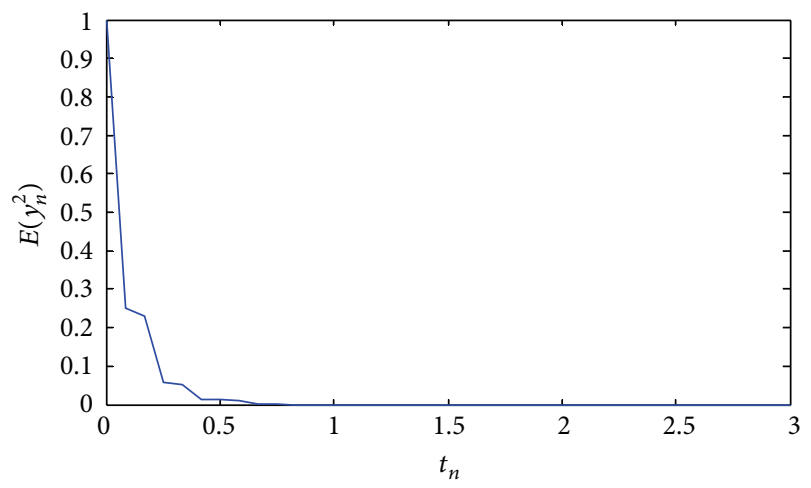

FIGURE 3: Mean square stability of SST method with $\theta=0.1$ and $\Delta t=0.1$

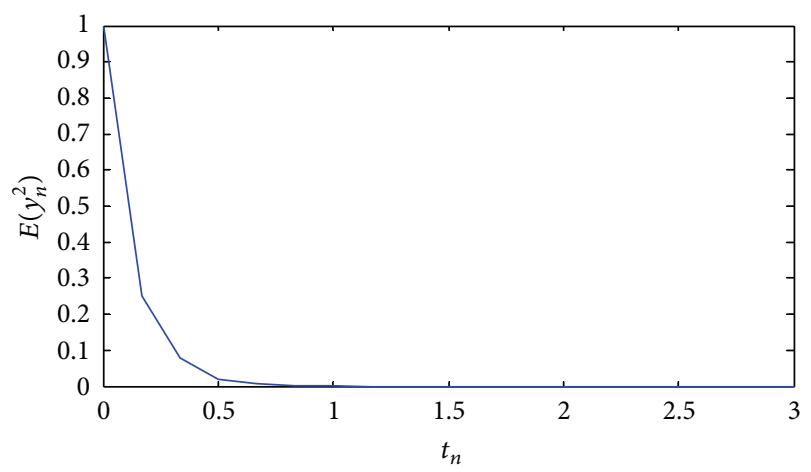

FIGURE 4: Mean square stability of SST method with $\theta=0.1$ and $\Delta t=0.2$.

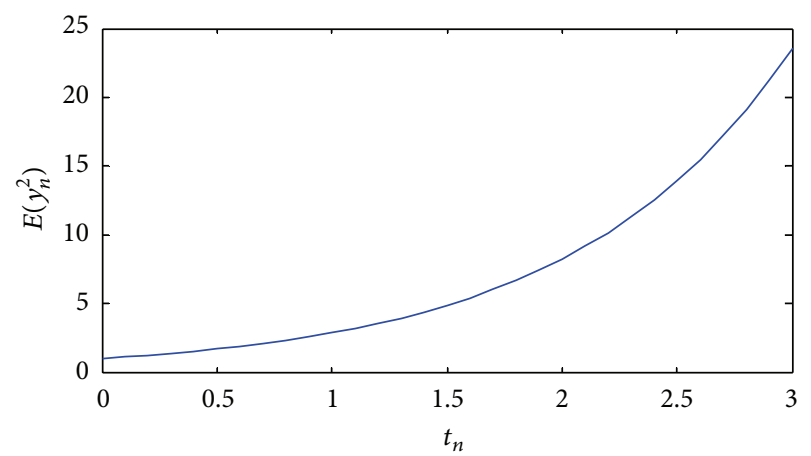

FIGURE 5: Unstable test for SST method with $\theta=0.1$ and $\Delta t=0.3$.

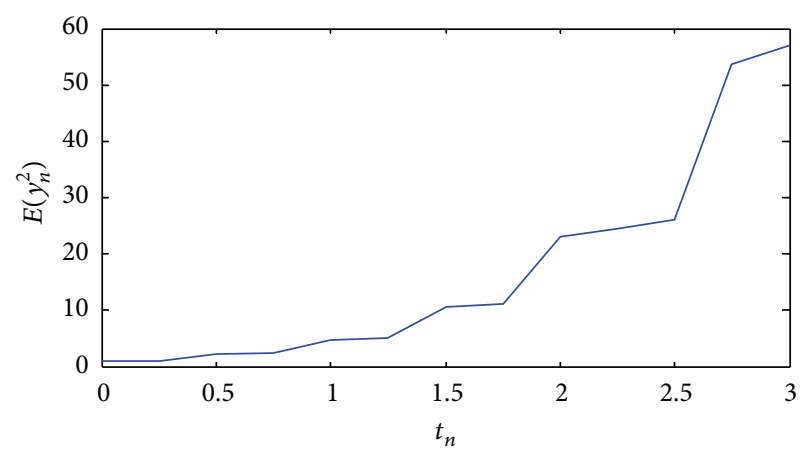

FIGURE 6: Unstable test for SST method with $\theta=0.1$ and $\Delta t=0.6$.

\section{Acknowledgments}

This work was supported by the Natural Science Foundation of Heilongjiang Province (A201418) and the Creative Talent Project Foundation of Heilongjiang Province Education Department (UNPYSCT-2015102).

\section{References}

[1] C. T. H. Baker and E. Buckwar, "Exponential stability in $p$ th mean of solutions, and of convergent Euler-type solutions, of stochastic delay differential equations," Journal of Computational and Applied Mathematics, vol. 184, no. 2, pp. 404-427, 2005.

[2] E. Buckwar, "The $\Theta$-maruyama scheme for stochastic functional differential equations with distributed memory term," Monte Carlo Methods and Applications, vol. 10, no. 3-4, pp. 235-244, 2004.

[3] E. Buckwar, "One-step approximations for stochastic functional differential equations," Applied Numerical Mathematics, vol. 56, no. 5, pp. 667-681, 2006.

[4] Y. Hu, S. A. Mohammed, and F. Yan, "Discrete-time approximations of stochastic delay equations: the Milstein scheme," The Annals of Probability, vol. 32, no. 1, pp. 265-314, 2004.

[5] M. Liu, W. Cao, and Z. Fan, "Convergence and stability of the semi-implicit Euler method for a linear stochastic differential delay equation," Journal of Computational and Applied Mathematics, vol. 170, no. 2, pp. 255-268, 2004.

[6] X. Mao, Stochastic Differential Equations and Their Applications, Harwood, Chichester, UK, 1997. 
[7] X. Mao, "Exponential stability of equidistant Euler-Maruyama approximations of stochastic differential delay equations," Journal of Computational and Applied Mathematics, vol. 200, no. 1, pp. 297-316, 2007.

[8] S. E. A. Mohammed, Stochastic Functional Differential Equations, Pitman, Boston, Mass, USA, 1984.

[9] V. B. Kolmanovskii and A. Myshkis, Applied Theory of Functional Differential Equations, Springer, Dordrecht, The Netherlands, 1992.

[10] F. Wu and X. Mao, "Numerical solutions of neutral stochastic functional differential equations," SIAM Journal on Numerical Analysis, vol. 46, no. 4, pp. 1821-1841, 2008.

[11] S. Zhou and F. Wu, "Convergence of numerical solutions to neutral stochastic delay differential equations with Markovian switching," Journal of Computational and Applied Mathematics, vol. 229, no. 1, pp. 85-96, 2009.

[12] S. Gan, H. Schurz, and H. Zhang, "Mean square convergence of stochastic $\theta$-Methods for nonlinear neutral stochastic differential delay equations," International Journal of Numerical Analysis and Modeling, vol. 8, no. 2, pp. 201-213, 2011.

[13] S. Zhou and Z. Fang, "Numerical approximation of nonlinear neutral stochastic functional differential equations," Journal of Applied Mathematics and Computing, vol. 41, no. 1-2, pp. 427445, 2013.

[14] B. Yin and Z. Ma, "Convergence of the semi-implicit Euler method for neutral stochastic delay differential equations with phase semi-Markovian switching," Applied Mathematical Modelling, vol. 35, no. 5, pp. 2094-2109, 2011.

[15] W. Wang and Y. Chen, "Mean-square stability of semi-implicit Euler method for nonlinear neutral stochastic delay differential equations," Applied Numerical Mathematics, vol. 61, no. 5, pp. 696-701, 2011.

[16] C. Huang, "Exponential mean square stability of numerical methods for systems of stochastic differential equations," Journal of Computational and Applied Mathematics, vol. 236, no. 16, pp. 4016-4026, 2012.

[17] X. Ding, Q. Ma, and L. Zhang, "Convergence and stability of the split-step $\theta$-method for stochastic differential equations," Computers \& Mathematics with Applications, vol. 60, no. 5, pp. 1310-1321, 2010.

[18] H. Zhang, S. Gan, and L. Hu, "The split-step backward Euler method for linear stochastic delay differential equations," Journal of Computational and Applied Mathematics, vol. 225, no. 2, pp. 558-568, 2009.

[19] J. Tan and H. Wang, "Convergence and stability of the splitstep backward Euler method for linear stochastic delay integrodifferential equations," Mathematical and Computer Modelling, vol. 51, no. 5-6, pp. 504-515, 2010.

[20] F. Jiang, Y. Shen, and J. Hu, "Stability of the split-step backward Euler scheme for stochastic delay integro-differential equations with Markovian switching," Communications in Nonlinear Science and Numerical Simulation, vol. 16, no. 2, pp. 814-821, 2011.

[21] C. Huang, "Dissipativity of Runge-Kutta methods for dynamical systems with delays," IMA Journal of Numerical Analysis, vol. 20, no. 1, pp. 153-166, 2000.

[22] C. Huang and Q. Chang, "Dissipativity of multistep RungeKutta methods for dynamical systems with delays," Mathematical and Computer Modelling, vol. 40, no. 11-12, pp. 1285-1296, 2004. 


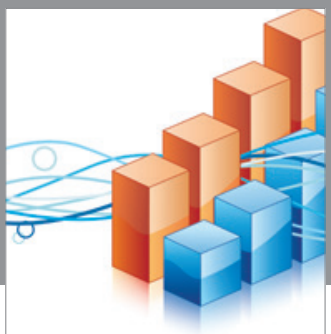

Advances in

Operations Research

vatem alat4

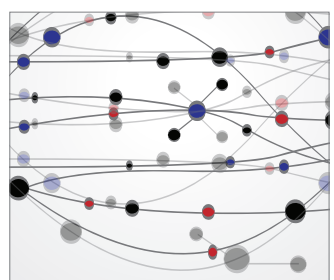

\section{The Scientific} World Journal
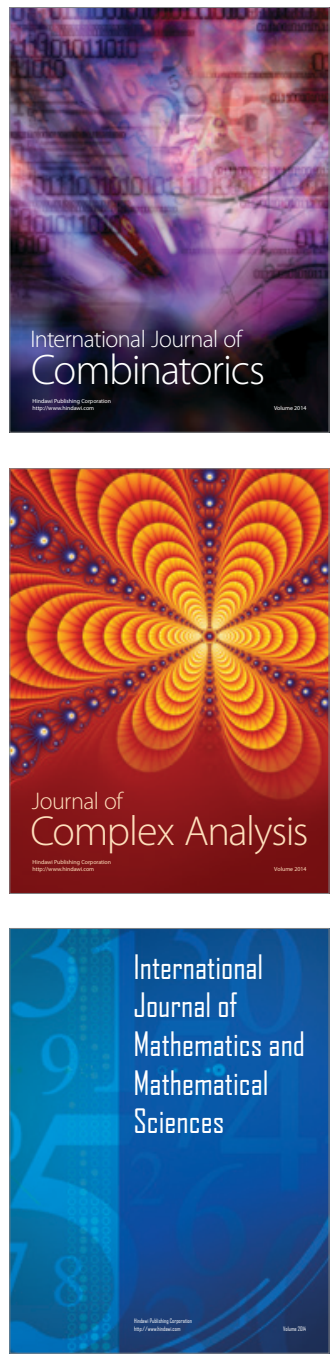
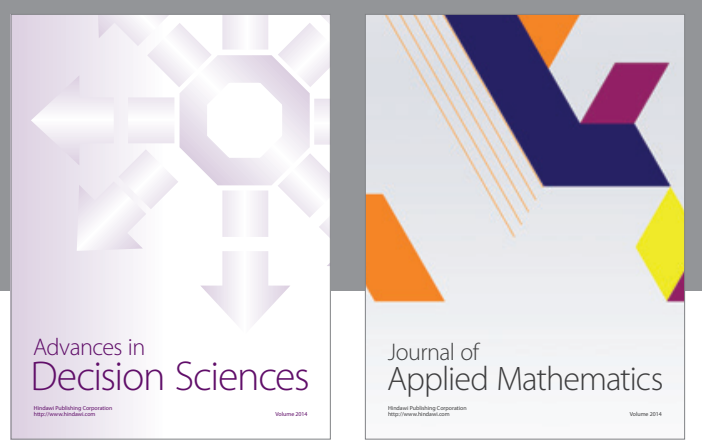

Algebra

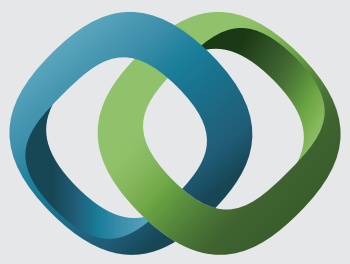

\section{Hindawi}

Submit your manuscripts at

http://www.hindawi.com
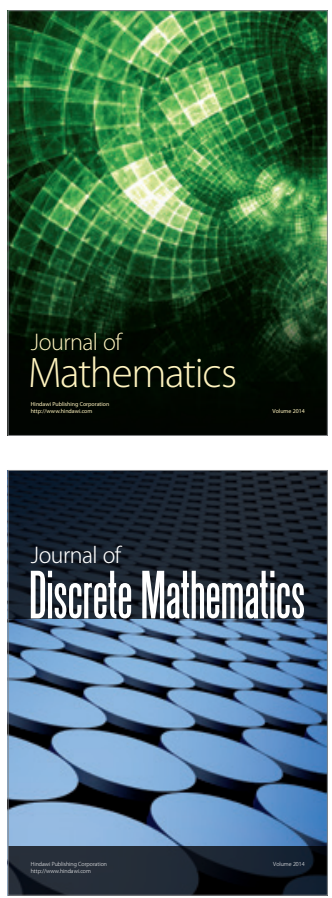

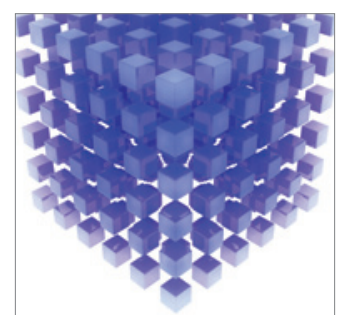

Mathematical Problems in Engineering
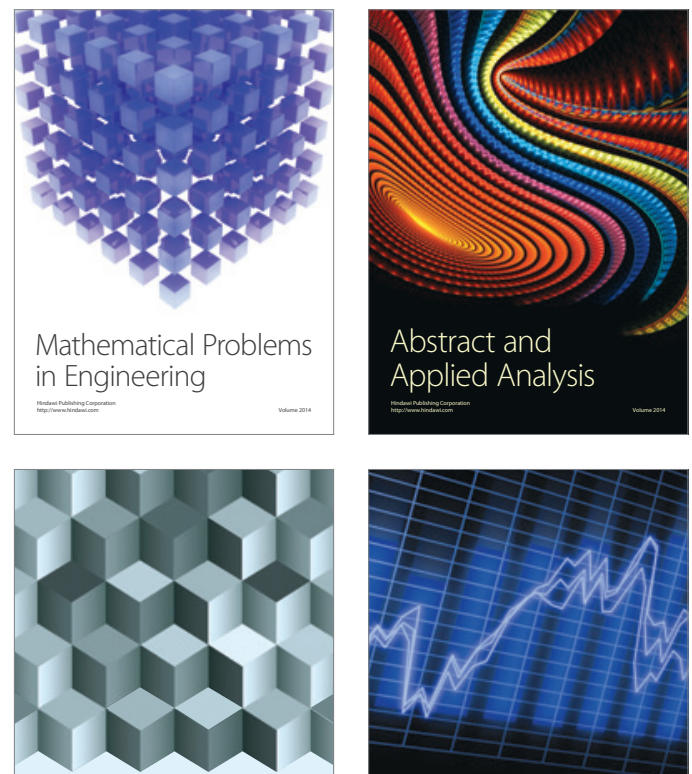

Journal of

Function Spaces

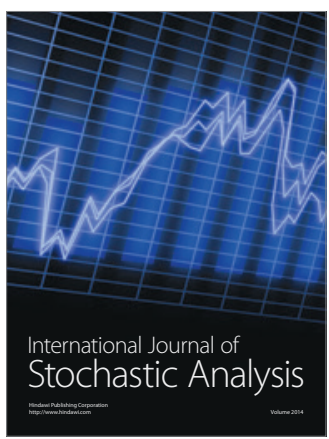

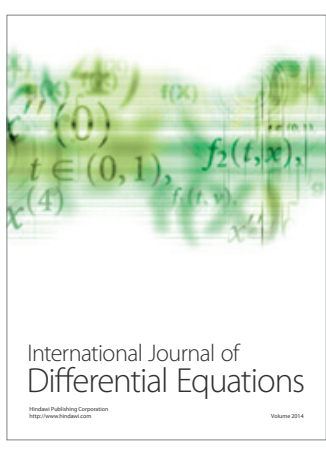
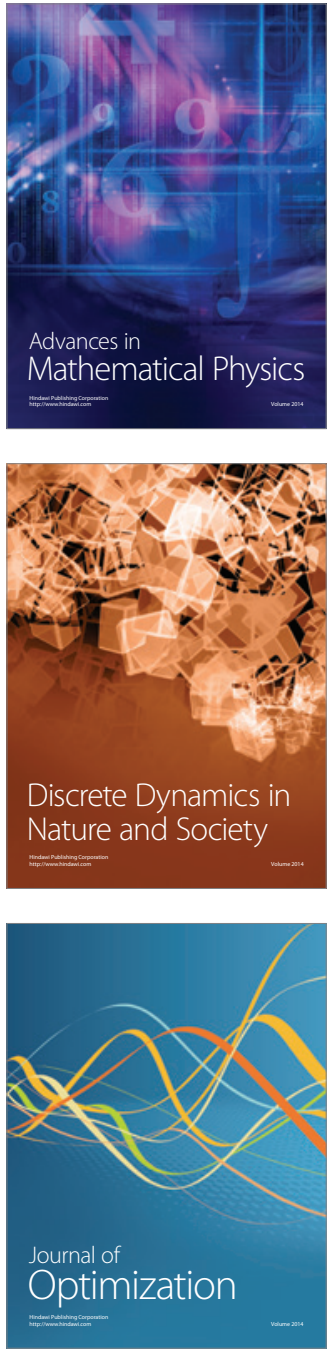